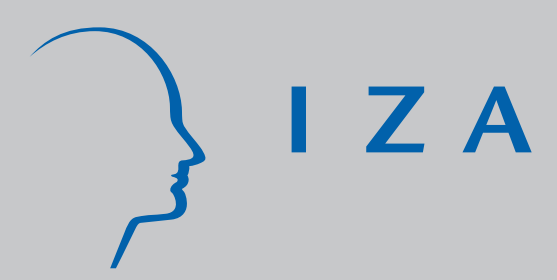

IZA DP No. 5900

Equilibrium Wage and Employment Dynamics in a Model of Wage Posting without Commitment

Melvyn G. Coles

Dale T. Mortensen

August 2011

Forschungsinstitut

zur Zukunft der Arbeit

Institute for the Study of Labor 


\title{
Equilibrium Wage and Employment Dynamics in a Model of Wage Posting without Commitment
}

\author{
Melvyn G. Coles \\ Unversity of Essex \\ Dale T. Mortensen \\ Northwestern University, Aarhus University, \\ NBER and IZA
}

\section{Discussion Paper No. 5900 \\ August 2011}

\author{
IZA \\ P.O. Box 7240 \\ 53072 Bonn \\ Germany \\ Phone: +49-228-3894-0 \\ Fax: +49-228-3894-180 \\ E-mail: iza@iza.org
}

\begin{abstract}
Any opinions expressed here are those of the author(s) and not those of IZA. Research published in this series may include views on policy, but the institute itself takes no institutional policy positions.

The Institute for the Study of Labor (IZA) in Bonn is a local and virtual international research center and a place of communication between science, politics and business. IZA is an independent nonprofit organization supported by Deutsche Post Foundation. The center is associated with the University of Bonn and offers a stimulating research environment through its international network, workshops and conferences, data service, project support, research visits and doctoral program. IZA engages in (i) original and internationally competitive research in all fields of labor economics, (ii) development of policy concepts, and (iii) dissemination of research results and concepts to the interested public.
\end{abstract}

IZA Discussion Papers often represent preliminary work and are circulated to encourage discussion. Citation of such a paper should account for its provisional character. A revised version may be available directly from the author. 
IZA Discussion Paper No. 5900

August 2011

\title{
ABSTRACT \\ Equilibrium Wage and Employment Dynamics in a Model of Wage Posting without Commitment
}

\begin{abstract}
A rich but tractable variant of the Burdett-Mortensen model of wage setting behavior is formulated and a dynamic market equilibrium solution to the model is defined and characterized. In the model, firms cannot commit to wage contracts. Instead, the Markov perfect equilibrium to the wage setting game, characterized by Coles (2001), is assumed. In addition, firm recruiting decisions, firm entry and exit, and transitory firm productivity shocks are incorporated into the model. Given that the cost of recruiting workers is proportional to firm employment, we establish the existence of an equilibrium solution to the model in which wages are not contingent on firm size but more productive employers always pay higher wages. Although the state space, the distribution of workers over firms, is large in the general case, it reduces to a scalar that can be interpreted as the unemployment rate in the special case of homogenous firms. Furthermore, the equilibrium is unique. As the dimension of the state space is equal to the number of firms types in general, an (approximate) equilibrium is computable.
\end{abstract}

JEL Classification: $\quad$ D21, D49, E23, J42, J64

Keywords: wage dispersion, wage setting, rank-preserving equilibrium

Corresponding author:

Dale T. Mortensen

Dept of Economics

Northwestern University

2001 Sheridan Road

Evanston, IL 60208

E-mail: d-mortensen@northwestern.edu

\footnotetext{
*We acknowledge valuable comments and suggestions from Robert Shimer and Philipp Kircher.
} 


\section{Introduction}

The model studied in this paper is one in which employers set the wage paid, in the tradition of Diamond (1971), Burdett and Judd (1983), Burdett and Mortensen (1998), Coles (2001) and Moscarini and Postel-Vinay (2010). It differs from these papers by introducing recruiting behavior at a cost of the form estimated by Merz and Yashiv (2007), firm entry and exit, and a transitory firm productivity shock. Like Moscarini and Postel-Vinay (2010) our purpose is to propose a rich but tractable dynamic variant of the Burdett-Mortensen model that can be used for both macro economic and policy applications. Following Coles (2001), the paper develops the implications of a sub-game perfect solution in Markov strategies to the wage setting game when firms cannot precommit to contracts that specify future wages. Interest in this case arises because the Burdett-Mortensen solution is not generally time consistent.

In the environment studied, there are many workers and firms, indeed a continuum of each. A firm can have many workers in the model. As is standard in this literature, every agent, worker and firm, is risk neutral and acts to maximize the expected present value of future income. Workers, employed as well as unemployed, randomly seek job vacancies that arise only sequentially. Each firm chooses a current wage and recruiting strategy and each worker follows a job acceptance and quit strategy that are respectively optimal given those of all other agents under rational expectations regarding the future evolution of the market state. For the specification of the cost of recruiting and hiring considered, one in which the cost is proportional to the number of employees, the wage paid and the hiring strategy pursued by any firm are both independent of firm size. This fact provides the simplification needed for tractability without violating the empirical relationships found in micro firm data.

In the language of Moscarini and Postel-Vinay (2010), a market equilibrium is rank-preserving if the rank of each firm's lifetime wage offer in the market wage offer distribution is its rank in the distribution of productivity at every point in time. In such an equilibrium, turnover is efficient in the sense that every worker moves from a less to a more productive firm when an opportunity arise. Although Moscarini and Postel-Vinay (2010) demonstrate the existence of a recursive rank-preserving equilibrium, restrictions on initial conditions are required. Specifically, because the wage strategy is size dependent in their model, higher paying firms must be larger initially. 
Unfortunately, this condition is violated in real data because firms die and new firms enter relatively small, independent of productivity. Although this possibility is incorporated in our model, their condition is not needed because a firm's size does not directly affect the optimal wage choice given the cost of hiring and recruiting function assumed.

Given the restrictions on primitives needed to guarantee the existence of bounded values for all agents in our model, we show that an essentially unique recursive rank-preserving equilibrium exists in the special case of equally productive firms. Formally, any equilibrium solution is isomorphic to the unique stable saddle path of a ordinary differential equation system that describes the adjustment dynamics of the value of a job-worker match and aggregate employment to their unique steady state values. In the case of firm heterogeneity with respect to productivity, we establish the existence of at least one rank preserving equilibrium when the distribution of firm productivity is approximated by a discrete distribution.

Menzio and Shi (2010) develop and study a recursive model of directed search that also allows for search on-the-job. In their paper, they suggest that directed search is a more useful approach for understanding labor market dynamics. They claim that models of random search in the BurdettMortensen tradition are intractable because the decision relevant state space is the evolving distribution of wages, which is of infinite dimension. Although the directed search model in arguably simpler in some respects, their principal objection to a random search model is simply not valid in the variant considered in the paper. The shape of the wage offer c.d.f. at any point in time is an equilibrium outcome of the wage and recruiting behavior of firms and the acceptance and quit strategy of worker with a location determined by the distribution of employment over firm types. Hence, at least as an approximation, the decision relevant state variable is a finite vector that characterizes this distribution. Indeed, in the special case of identical firms, the state variable is simply the aggregate level of employment.

A troublesome implication of the original Burdett-Mortensen model for empirical implementation is that the implied equilibrium offer and wage distributions are convex while in the data they are unimodal and well approximated by the log normal (See Christiansen et al. (2005)). Although a unimodal distribution is possible when firms differ in labor productivity, Mortensen (2003) shows that model is not consistent with both the firm wage distribution and the distribution of firm productivity in Danish data. The theoretical distribution of productivity which is consistent with the wage 
distribution is far more skewed to the right than that observed. The inconsistency reflects the fact that high wage firms have much less incentive to compete for workers by paying a higher wage than do low wage firms because workers only move up the wage ladder.

The Coles (2001) solution to the wage setting game, supported by optimal quit behavior of employees, is such that every firm is indifferent between offering a high wage and the workers' common reservation wage. Like the Burdett-Mortensen model, firms of equal productivity do pay different wages. However, the high wage which is actually paid in equilibrium is simply equal to the saving in turnover costs that would have to be incurred where the reservation wage paid instead. In the special case in which recruiting costs are primarily those associated with processing applications and training workers rather than advertising the job opening, this condition implies that the equilibrium distribution of offers is uniformly distributed over the set of firms that are equally productive. Furthermore, the offer density is decreasing when firms differ in productivity. Finally, the properties of the employment weighted distribution of wage, a distribution which is stationary only in steady state, can be quite complex.

\section{The Model}

\subsection{Specification}

Time is continuous, has an infinite horizon and all discount the future at rate $r>0$. The labor market is populated by a unit measure of equally productive, risk neutral immortal workers. Every worker is either unemployed or employed, earns a wage if employed, and the value of home production, $b \geq 0$, if not. There is also a measure of risk neutral, heterogeneous firms. Market output is produced by matched workers and firms with a linear technology.

New firms enter at rate $\mu>0$, continuing firms die at rate $\delta>0$ so that the measure of firms is stationary and equal to $\mu / \delta$. At entry, the productivity of a new firm $p$ is determined as a random draw from the c.d.f. $\Gamma_{0}($.$) .$ Continuing firms are subject to a technology jump process characterized by a given arrival rate $\gamma \geq 0$ and a distribution of new values $\Gamma_{1}($.$) . The common$ support of both distributions is $[b, \bar{p}]$ and, for ease of exposition, $\Gamma_{0}, \Gamma_{1}$ are continuous functions (no mass points). As in Klette and Kortum (2005) and Lentz and Mortensen (2010), one can think of the entry flow as firms with 
new products and the exit flow as firms that are destroyed because their product is no longer in demand.

Given the above productivity and turnover processes, it is a straightforward algebraic exercise to compute the stationary distribution of firm productivity $\Phi(p)$ and show that it is a continuous and increasing function over $[b, \bar{p}]$. It is convenient, however, to instead rank firms by their productivity; i.e. a firm with productivity $p$ is equivalently described as having rank $x \in[0,1]$ solving $x=\Phi(p)$. Defining $p(x)=\Phi^{-1}(x)$, each firm is then characterized by $(x, n ; s)$ where $x$ describes its rank (with corresponding productivity $p=p(x)$ ), $n$ describes its (integer) number of employees and $s$ the aggregate state vector.

Throughout we only consider stationary (Markov) equilibria where the market state process $\left\{s_{t}\right\}$ is Markov and known to all agents. As all agents are small, each takes this process as given. At time $t$ in state $s=s_{t}$, firm $(x, n ; s)$ generates expected discounted profit $\Pi(x, n ; s)$ by paying wage $w=$ $w(x, n ; s)$ to each of its employees and by recruiting additional employees at an expected rate $H=H(x, n ; s) . W(x, n ; s)$ denotes a worker's expected lifetime wage when employed by such a firm while $V_{u}(s)$ denotes the expected value of being unemployed. Below we will derive the information relevant state from the structure of the model. To fix ideas, however, it is useful to define $A_{t}(x, n)$ as the distribution of employment across firms in the market at time $t$. In what follows, this distribution function can be considered as the market state variable $s_{t}$. Given this state variable at time $t$ and the wage strategies of firms, one can then compute $G_{t}(W)$ defined as the date $t$ proportion of workers who enjoy lifetime value at least as great as $W$. As no employee finds $W<V_{u}$ acceptable, $G_{t}\left(V_{u}\right)$ then describes the date $t$ unemployment rate. Finally for $W \geq V_{u}, G_{t}(W)-G_{t}\left(V_{u}\right)$ is the date $t$ proportion of workers who are employed with lifetime value $W$ or less.

New firms enter with a single worker, the innovator. Once a new firm enters, the innovator sells the firm to risk neutral investors for its value and reverts to his/her role as a worker. Each firm faces costs of expanding its labour force. Suppose firm $(x, n ; s)$ posts $Y$ vacancies and, with a random contact technology, let $\eta(s) Y$ describe the rate at which this firm contacts potential new employees. As employment at the firm yields worker value $W=W(x, n ; s)$ then $G_{t}(W)$ is the probability that a randomly contacted worker is willing to accept the job offer. Thus to hire a new employee at rate $H$ the firm must post $Y$ vacancies satisfying $H=\eta(s) G_{t}(W) Y$. Note that firms which pay lower wages need to post more vacancies to achieve a given 
$H$.

There are two costs to the recruiting and hiring workers. First $k Y$ describes the direct cost of posting $Y$ vacancies to attract the required number of hires. Second and perhaps more importantly, vetting job applicants and training new hires is costly. Let $H / n$ describe the firm's recruitment effort required per employee. If each employee contributes to the recruiting process equally, the cost of hiring at rate $H$ is $n c(H / n)$ where $c($.$) describes the per$ employee cost. ${ }^{1}$ The cost of recuriting at rate $H$ at firm $(x, n ; s)$ is then

$$
\frac{k H}{\eta(s) G_{t}(W)}+n c(H / n)
$$

where we $c($.$) is increasing and strictly convex by assumption.$

At rate $\mu$ each worker, whether employed or unemployed, conceives a new business idea and so has the opportunity to start-up a new firm. We assume the worker always chooses to accept the opportunity and so $\mu$ describes the entry rate of new firms. This of course requires that an employed worker always prefers to quit to start-up a new firm rather than remain employed with its existing firm, a condition which holds under the assumption that new firms are sufficiently more productive than continuing firms. Of course, this requirement holds automatically when each new product is more valuable than the one it replaces as in the model of Klette and Kortum (2004). ${ }^{2}$

Finally, given firm $(x, n ; s)$ offers value $W=W(x, n ; s)$, employees quit at rate $q(W ; s)=\mu+\lambda(s)[1-F(W ; s)]$ where $\mu$ is the rate of firm creation per employee, $\lambda(s)$ is the job offer arrival rate per employee, and $F(W ; s)$ is the probability that any outside job offer is less than or equal to $W$. These objects are determined endogenously in equilibrium.

\subsection{The Wage Setting Game}

With precommitment at date $t=0$ on the entire path of future wages, Moscarini and Postel-Vinay (2010) construct Markov Perfect Equilibria to the extended Burdett and Mortensen (1998) framework [BM from now on] where wage strategies are conditioned on initial firm size. Here we assume

\footnotetext{
${ }^{1}$ This formulation of costs of adjustment is standard in the literature. As a case in point, see Merz and Yashiv (2007).

${ }^{2}$ This restriction is made for simplicity. Were it not so, then the entry decision is endogenous to the process under study. Adding this complication is both realistic and worth pursuing but goes beyond the scope of this paper.
} 
no precommitment on future wages and consider the continuous time limit $d t \rightarrow 0^{+}$. Suppose in the model described above that a Markov Perfect Equilibrium exists in which the equilibrium wage strategy of firm $(x, n ; s)$ is to set wage $w=w(x, n ; s)$ with probability one (independent of the previous history). Further suppose this wage strategy yields lifetime value $W(x, n ; s)>V_{u}(s)$ to its employees. Consider then the following wage deviation - the firm sets wage $w=0$. In the subgame (after a $d t>0$ delay), the firm's strategy implies it reverts with probability one to the equilibrium wage $w^{\prime}=w\left(x^{\prime}, n^{\prime} ; s^{\prime}\right)$. But as $d t \rightarrow 0$, Coles (2001) argues this wage deviation is always profitable. The reason is transparent in the steady state case: if employees at the highest wage firm always expect wage $w=\bar{w}$, their quit strategy is unchanged if the firm (only momentarily) announces $w=0$. But this cannot describe (subgame perfect) equilibrium behavior as the firm can then profitably deviate by (always momentarily) announcing $w=0 .^{3}$

The dynamic inconsistency in BM is that once a firm has grown to be large then, rather than announce a high wage $w$, it is strictly better off by announcing the worker's reservation wage $R$ (to extract incumbent employee search rents). Coles (2001) identifies a dynamically consistent (Markov) equilibrium where instead each firm $(x, n ; s)$ has two optimal strategies: the equilibrium wage path $w=w(x, n ; s)$, which we will refer to as the high wage path, and the rip-off strategy $w=R(x, n ; s)$ which leaves each employee indifferent to quitting into unemployment (but does not do so by convention). The firm's equilibrium (Markov) pricing strategy (for $d t>0$ but arbitrarily small) is:

(a) if last period the firm announced wage $w^{\prime} \geq w\left(x^{\prime}, n^{\prime} ; s^{\prime}\right)$ then announce $w=w(x, n ; s)$;

(b) if last period the firm announced wage $w^{\prime}<w\left(x^{\prime}, n^{\prime} ; s^{\prime}\right)$ then announce $w=R(x, n ; s)$.

Note the critical property: if the firm ever announces a wage below the high wage path $w(x, n ; s)$, employees expect the rip-off strategy thereafter.

\footnotetext{
${ }^{3}$ The argument clearly extends to mixed strategies. It essentially establishes there cannot be a Markov perfect equilibrium with $W>V_{u}$ (any firm will deviate to $w=0$ ). A MPE exists if it is assumed workers cannot quit until the end of the $d t>0$ period: in that case the unique MPE is that all firms pay $w=b$ and there is no quit turnover. Conversely if instead workers can quit at the start of the period and all other firms offer $w=b$, then $w=b+\varepsilon$ is a profitable deviation. As the only candidate MPE is that $W=V_{u}$, a MPE cannot then exist. We believe the latter scenario is the more interesting case as it presumes firms can precommit to a higher wage to attract new employees, though only for a (very) limited period.
} 
This switch in employee expectations on future wages generates a sufficiently large change in turnover behavior that setting wage below $w(x, n ; s)$ is never profit increasing. Specifically the arguments developed in Coles (2001) establish for $d t>0$ but arbitrarily small:

(i) announcing a deviating higher wage $w^{\prime}>w(x, n ; s)$ has such a small impact on turnover that offering $w^{\prime}=w(x, n ; s)$ is strictly more profitable;

(ii) announcing $w^{\prime} \in(R, w(x, n ; s))$, which implies workers expect the wage $w=R$ in the entire future, has such a small impact on turnover that offering $w^{\prime}=R$ is strictly more profitable; while

(iii) offering $w^{\prime}<R$, say $w^{\prime}=0$, implies all workers quit (they anticipate $w=R$ in the entire future) and so yields zero profit.

Coles (2001) shows this equilibrium (as $d t \rightarrow 0$ ) yields the original (steady state) outcome described in BM when the discount rate $r$ is arbitrarily small. The approach is particularly useful here for two reasons. First the equilibrium wage path $w=w(x, n ; s)$ remains Markov and so can be embedded into a non-steady state framework. Second it yields two key simplifications:

(i) the rip-off strategy $w=R$ implies each firm with $p(x)>R$ and $n \geq 1$ must make strictly positive profit;

(ii) for all $x$, the high wage path implies $W(x, n ; s) \geq V_{u}{ }^{4}$ Thus unemployed workers and workers employed at firms using the rip-off strategy will accept the first (outside) job offer received.

\subsection{The Reservation Wage}

Because individual workers are hired and quit sequentially, the number of employees in a continuing firm is a stochastic process. Indeed, $n$ is a birthdeath process with an absorbing state that occurs when the firm dies. That is, the firm's labor force size is an integer that can only transit from the value $n$ to $n+1$ if a worker is hired, from $n$ to $n-1$ if a worker quits, or to zero if the firm loses its market in any sufficiently short time period of length $d t>0$. The transition rates for these three events are respectively the hire frequency $H(x, n ; s)$, the quit frequency $n q(W ; s)$, and the destruction frequency $\delta$.

Define $\widehat{\Gamma_{0}}(x)=\Gamma_{0}(p(x))$ and $\widehat{\Gamma_{1}}(x)=\Gamma_{1}(p(x))$ for $x \in[0,1]$. Consider a worker in firm $(x, n ; s)$ that follows the equilibrium high wage path $w(x, n ; s)$. The value of employment or lifetime wage $W(x, n ; s)$ solves the recursive

\footnotetext{
${ }^{4} W<V_{u}$ generates zero profit and this strategy is then dominated by the ripoff strategy (strictly if $p(x)>b$ ).
} 
equation:

$$
\begin{aligned}
r W(x, n ; s)= & w(x, n ; s)+\delta\left[V_{u}(s)-W(x, n ; s)\right] \\
& +\gamma \int_{0}^{1}[W(z, n ; s)-W(x, n ; s)] d \widehat{\Gamma_{1}}(z) \\
& +\lambda(s) \int \max [X-W(x, n ; s), 0] d F(X ; s) \\
& +\mu \int_{0}^{1}[\Pi(z, 1 ; s)+W(z, 1 ; s)-W(x, n ; s)] d \widehat{\Gamma_{0}}(z) \\
& +(n-1) q(W(x, n ; s) ; s)[W(x, n-1 ; s)-W(x, n ; s)] \\
& +H(x, n ; s)[W(x, n+1 ; s)-W(x, n ; s)]+\frac{\partial W}{\partial t} .
\end{aligned}
$$

In other words, the flow value of employment is equal to the wage income plus the expected capital gain associated with the possibility of firm destruction, a firm specific productivity shock, being offered a better job, creating a business start-up, a quit by another employee, a new hire, and the passage of time, all conditional on the market state $s$. The latter term captures the effect on $W$ through the non-steady state evolution of $s$ over time.

The equilibrium construction requires the firm is everywhere indifferent between sticking to the high wage path or deviating (in perpetuity) to the ripoff strategy $w=R(x, n ; s)$ where $R($.$) is the wage at which an employee is just$ indifferent to quitting into unemployment. Now the value of unemployment $V_{u}(s)$ solves the analogous equation

$$
\begin{aligned}
r V_{u}(s)= & b+\mu \int_{0}^{1}\left[\Pi(z, 1 ; s)+W(z, 1 ; s)-V_{u}(s)\right] d \widehat{\Gamma_{0}}(z) \\
& +\lambda(s) \int\left[X-V_{u}(s)\right] d F(X ; s)+\frac{\partial V_{u}}{\partial t} .
\end{aligned}
$$

Should the employer deviate by instead paying the worker's reservation wage 
in every state, the worker enjoys deviating lifetime value:

$$
\begin{aligned}
r W^{d}(x, n ; s)= & R(x, n ; s)+\delta\left[V_{u}(s)-W^{d}(x, n ; s)\right] \\
& +\gamma \int_{0}^{1}\left[\max \left\langle W^{d}(z, n ; s), V_{u}(s)\right\rangle-W^{d}(x, n ; s)\right] d \widehat{\Gamma_{1}}(z) \\
& +\lambda(s) \int\left[\max \left\langle X, W^{d}(x, n ; s)\right\rangle-W^{d}(x, n ; s)\right] d F(X ; s) \\
& +\mu \int_{0}^{1}\left[\Pi(z, 1 ; s)+W(z, 1 ; s)-W^{d}(x, n ; s)\right] d \widehat{\Gamma_{0}}(z) \\
& +(n-1) q\left(W^{d}(x, n ; s) ; s\right)\left[W^{d}(x, n-1 ; s)-W^{d}(x, n ; s)\right] \\
& +H^{d}(x, n ; s)\left[W^{d}(x, n+1 ; s)-W^{d}(x, n ; s)\right]+\frac{\partial W^{d}}{\partial t},
\end{aligned}
$$

where $H^{d}($.$) describes the firm's optimal recruitment rate given the rip-off$ wage strategy. As the construction of equilibrium requires $W^{d}(x, n ; s)=$ $V_{u}(s)$ for all $(x, n ; s)$, then substituting out $W^{d}$ in the latter expression and comparing with the Bellman equation for $V_{u}$ implies that the reservation wage,

$$
R(x, n ; s)=b,
$$

is the value of home production in all circumstances.

\subsection{The Size Independent Wage and Recruiting Policy}

We now establish that the equilibrium wage strategy is size independent. In the case of an operating firm with $n \geq 1$ employees the value of this firm $(x, n ; s)$ is defined recursively by

$$
(r+\delta) \Pi(x, n ; s)=\max _{H \geq 0}\left\langle\begin{array}{c}
(p(x)-w(x, n ; s)) n-\frac{k H}{\eta(s) G_{t}(W)}-n c(H / n) \\
+H[\Pi(x, n+1 ; s)-\Pi(x, n ; s)] \\
+n[\mu+q(W ; s)][\Pi(x, n-1 ; s)-\Pi(x, n ; s)] \\
+\gamma \int_{0}^{1}(\Pi(z, n ; s)-\Pi(x, n ; s)) d \widehat{\Gamma_{1}}(z)+\frac{\partial \Pi}{\partial t}
\end{array}\right\rangle
$$

where the right hand side contains the current cash flow, the return to recruiting an additional employee, the cost of losing an employee, the likelihood of a productivity shock and the passage of time respectively.

The equilibrium construction requires the firm is indifferent to the rip-off strategy which, by equation (3), is to set $w=b$ in perpetuity (and thus yield employee value $V_{u}(s)$ ). The value of this deviating strategy is therefore: 


$$
(r+\delta) \Pi^{d}(x, n ; s)=\max _{H^{d} \geq 0}\left\langle\begin{array}{c}
(p(x)-b)) n-\frac{k H^{d}}{\eta(s) G_{t}\left(V_{u}\right)}-n c\left(H^{d} / n\right) \\
+H^{d}\left[\Pi^{d}(x, n+1 ; s)-\Pi^{d}(x, n ; s)\right] \\
+n[\mu+\lambda(s)]\left[\Pi^{d}(x, n-1 ; s)-\Pi^{d}(x, n ; s)\right] \\
+\gamma \int_{0}^{1}\left(\Pi^{d}(z, n ; s)-\Pi^{d}(x, n ; s)\right) d \widehat{\Gamma_{1}}(z)+\frac{\partial \Pi^{d}}{\partial t}
\end{array}\right\rangle
$$

where $\lambda(s)$ is the employee quit rate as each leaves to their first outside job offer. Define $h^{d}=H^{d} / n$ which is recruitment effort per employee in the deviating strategy. ${ }^{5}$ It is immediate that the solution to this equation is $\Pi^{d}(x, n ; s)=n v(x ; s)$ where $v(x ; s)$, which describes the value of each employee, solves

$$
(r+\delta) v(x ; s)=\max _{h^{d} \geq 0}\left\langle\begin{array}{c}
p(x)-b-\frac{k h^{d}}{\eta(s) G_{t}\left(V_{u}\right)}-c\left(h^{d}\right) \\
+\left[h^{d}-\mu-\lambda(s)\right] v(x ; s) \\
+\gamma \int_{0}^{1}(v(z ; s)-v(x ; s)) d \widehat{\Gamma_{1}}(z)+\frac{\partial v}{\partial t}
\end{array}\right\rangle
$$

As $\Pi(x, n ; s)=\Pi^{d}(x, n ; s)=n v(x ; s)$, then the Bellman equation for $\Pi($. reduces to:

$$
(r+\delta) v(x ; s)=\max _{h \geq 0}\left\langle\begin{array}{c}
p(x)-w(x, n ; s)-\frac{k h}{\eta(s) G_{t}(W)}-c(h) \\
+[h-\mu-q(W ; s)] v(x ; s) \\
+\gamma \int_{0}^{1}(v(z ; s)-v(x ; s)) d \widehat{\Gamma_{1}}(z)+\frac{\partial v}{\partial t}
\end{array}\right\rangle .
$$

As $v($.$) is size independent it is immediate that the equilibrium wage w($. must also be independent of size $n$. With a slight abuse of notation, we now write $w=w(x ; s)$ and note the lifetime value of employment must also be size independent; i.e. $W=W(x ; s)$.

Before simplifying the model further, it is important to note this sizeindependence result is not solely a consequence of the assumed cost structure $c($.$) . At this stage we could set c($.$) to zero, so that the only cost to$ hiring workers is the standard vacancy posting cost, and the size independence result remains. The essential difference to previous work is that here firms can directly control the hiring margin with a recruitment strategy $H$, leaving the firm's wage strategy to control its employee quit rate. Indeed we

\footnotetext{
${ }^{5}$ by assumption the firm using the rip-off strategy can recruit unemployed workers as $V_{u} \leq W^{d}$.
} 
show below that the equilibrium wage paid depends only on the return to reducing employee quit rates. Instead when wages are used to control both the recruitment rate of new employees and the quit rate of existing employees, as in BM, Coles (2001) Moscarini and Postel-Vinay (2010), the resulting equilibrium structure of wages is very different. We discuss this issue further in the Conclusion.

\subsection{A Baseline Case}

The model simplifies if the cost of posting a vacancy, $k$, is trivial. The cost of adjustment function estimated by Merz and Yashiv (2007) provides empirical support for the relevance of this case. In the notation of this model, they estimate a cost of recruiting and hiring function of the form $\left(a h+c_{0} h^{c_{1}}\right) n$, which is a special case of that specified above since the hire rate is linear in the number of vacancies posted. Although the estimates of the non-linear component play a large role in explaining the dynamics of factor adjustment as well as the ability of their model to explain firm market values, their point estimate of the linear component is slightly negative and statistically insignificant. Hence, the restriction $k=0$ is consistent with their findings. Furthermore, this evidence that labor adjustment costs are primarily processing and training costs rather then those associated with advertising seems to be consistent with everyday experience.

Define the recruitment effort function as

$$
h^{*}(v)=\arg \max _{h \geq 0}[h v-c(h)] .
$$

When $k=0$, the optimal equilibrium and deviating recruiting policies are the same, both given by $h(x ; s)=h^{*}(v()$.$) . Furthermore equations (4),(5)$ imply

$$
\begin{aligned}
w(x ; s) & =b+[\lambda(s)-q(W(x ; s) ; s)] v(x ; s) \\
& =b+\lambda(s) F(W(x ; s) ; s) v(x ; s)
\end{aligned}
$$

as $q(W ; s)=\mu+\lambda(s)[1-F(W ; s)]$. Note the wage premium $w-b$ equals the saving through the reduced quit flow attributable to paying the higher wage. The value of each employee to firm $(x, n ; s)$ is then the solution to the first order differential equation

$$
(r+\delta+\gamma+\mu+\lambda(s)) v(x ; s)-\dot{v}=\max _{h \geq 0}\left\{\begin{array}{c}
p(x)-b+\gamma \int_{0}^{1} v(z ; s) d \widehat{\Gamma_{1}}(z) \\
-c(h)+h v(x ; s)
\end{array}\right\} .
$$


where the dot refers to the time derivative $\partial v / \partial t$ and captures the dynamics due to the non-steady state evolution of $s$. The analysis presented in the rest of the paper is restricted to this case.

\section{Rank Preserving Equilibria}

\subsection{Comparative Dynamics}

A wage policy function $w(x ; s)$ that is strictly increasing in $x$ for every state of the market $s$, is said to be rank-preserving in the sense that the rank of a firm's wage in the wage offer distribution is the same as its rank in the productivity distribution in every market state. As workers voluntarily move only from worse to better paying firms, a market equilibrium is rankpreserving in the sense of Moscarini and Postel-Vinay if the equilibrium wage policy function is rank preserving.

We first establish that $v($.$) is increasing in x$; i.e. higher productivity firms enjoy higher employee values.

Proposition 1 The value of an employee $v(x ; s)$ is unique, continuous and increasing in $x$ for every $s$.

Proof. Any solution $v(x ; s)$ to $(7)$ is a fixed point of the contraction

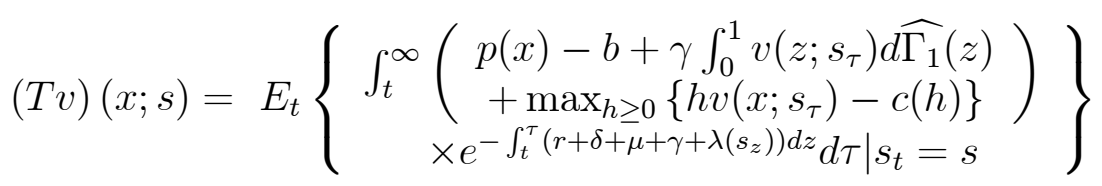

where $E_{t}$ denotes the expectation with respect to information available at time $t$. Given that $(T v)(x ; s)$ is continuous and increasing in $x$ if $v(x ; s)$ has these properties, $T$ maps the space of bounded, continuous and increasing functions in $x$. Hence a fixed point of the map exists, is unique and has the property stated.

Anticipating that higher productivity firms have a greater marginal willingness to pay for a lower quit rate, we focus on rank-preserving equilibria where $w(x ; s)$ is indeed increasing in $x$. Given higher productivity firms pay higher wages, it is immediate that lifetime values $W(x ; s)$ are also increasing in $x$. As both $v($.$) and W($.$) are increasing in x$, the wage equation (6) confirms that the equilibrium wage $w($.$) increases with x$. 
A rank-preserving equilibrium yields the following simplification: that $\widehat{F}(x ; s) \equiv F(W(x ; s) ; s)$ describes both the fraction of job offers made by firms with type no greater than $x$ and also the fraction of job offers which have value no greater than $W(x ;$.$) . By further defining the worker surplus$ function $\sigma(x ; s)=W(x ; s)-V_{u}(s)$, equations (1) and (2) imply:

$$
\begin{aligned}
& (r+\delta+\mu+\gamma+\lambda(s)) \sigma(x ; s)-\dot{\sigma} \\
= & w(x ; s)-b+\gamma \int_{0}^{1} \sigma(z ; s) d \widehat{\Gamma_{1}}(z)+\lambda(s) \int_{0}^{x} \sigma_{z}(z ; s) \widehat{F}(z ; s) d z
\end{aligned}
$$

where $\sigma_{z}(z ; s) \equiv \partial \sigma / \partial z$.

Proposition 2 If $w(x ; s)$ is continuous and increasing in $x$ for every $s$, then the surplus value of employment, $W(x ; s)-V_{u}(s)$ is unique, positive, continuous and increasing in $x$.

Proof. Any forward solution to equation (8) is a fixed point of the continuous and increasing contraction $T$ defined by

$$
(T \sigma)(x ; s)=E_{t}\left\{\int_{t}^{\infty}\left(\begin{array}{c}
w\left(x ; s_{\tau}\right)-b+\gamma \int_{0}^{1} \sigma\left(z ; s_{\tau}\right) d z \\
+\lambda\left(s_{\tau}\right) \int_{0}^{x} \widehat{F}\left(z ; s_{\tau}\right) \sigma_{z}\left(z ; s_{\tau}\right) d z \\
\times e^{-\int_{t}^{\tau}\left(r+\delta+\mu+\gamma+\lambda\left(s_{z}\right)\right) d z} d \tau
\end{array}\right\}\right.
$$

Obviously, $T$ maps the set of bounded, increasing and continuous functions of $x$ into itself under the hypothesis. Hence, any fixed point of the map is unique has the property.

\subsection{Aggregation}

From now on we restrict attention to optimal wage policy functions which are rank-preserving and confirm the existence and uniqueness of such a policy as part of the characterization of the equilibrium. This section aggregates across the wage offer and hiring strategies of firms and so identifies job offer propensities $\lambda(s), \widehat{F}(. ; s)$ consistent with a rank-preserving equilibrium.

Recall that each firm employs an integer number of workers. Suppose at date $t$, the firm of rank $x$ has $n(x, t)$ employees. In the limiting case of a continuum of firms and by the law of large numbers, $N_{t}(x)=\int_{0}^{x} n(z, t) d z$ 
describes the total number of workers employed at firms with type no greater than $x$.

Suppose now in state $s=s_{t}$ at date $t$, firm $(x, n ; s)$ adopts optimal recruitment rule $H=h(x ; s) n$ where $h=h^{*}(v()$.$) . Recall that to hire at this$ expected rate, the matching process requires the firm posts $Y(x, n ; s)$ vacancies where $\eta(s) G_{t}(W(x ; s)) Y=H$. Thus optimal vacancy posting implies

$$
\eta(s) Y(x, n ; s)=\frac{h(x ; s) n}{G_{t}(W(x ; s))} .
$$

Integrating across all firms with rank no greater than $x$ yields aggregate job offer rate

$$
\int_{0}^{x} \eta(s) Y(z, n(z, t) ; s) d z=\int_{0}^{x} \frac{h(z ; s)}{G_{t}(W(z ; s))} d N_{t}(z) \forall x \in[0,1] .
$$

If $U_{t}$ denotes the number of unemployed workers at time $t$, a rank preserving equilibrium further implies

$$
G_{t}(W(z ; s))=U_{t}+N(z, t) .
$$

Thus we obtain aggregate job offer rate:

$$
\int_{0}^{x} \eta(s) Y(z, n(z, t) ; s) d z=\int_{0}^{x} \frac{h(z ; s) d N_{t}(z)}{U_{t}+N_{t}(z)} \forall x \in[0,1] .
$$

As job offers are random and there is a unit mass of workers, the arrival rate of a job offer to any given worker must therefore be

$$
\begin{aligned}
\lambda(s) & \equiv \int_{0}^{1} \eta(s) Y(z, n(z, t) ; s) d z \\
& =\int_{0}^{1} \frac{h(z ; s) d N_{t}(z)}{U_{t}+N_{t}(z)}
\end{aligned}
$$

while the arrival rate of a job offer from a firm with type no greater than $x$ is

$$
\begin{aligned}
\lambda(s) \widehat{F}(x ; s) & \equiv \int_{0}^{x} \eta(s) Y(z, n(z, t) ; s) d z \\
& =\int_{0}^{x} \frac{h(z ; s) d N_{t}(z)}{U_{t}+N_{t}(z)} .
\end{aligned}
$$




\subsection{Definition of Equilibrium}

In principle the aggregate state variable $s_{t}$ at time $t$ is the distribution of firm sizes for each type $x$, which we previously denoted $A_{t}(x, n)$. For each firm $(x, n ; s)$, however, both the wage strategy $w(x ; s)$ and recruitment effort strategy $h(x ; s)$ are firm size independent. In what follows we show $N_{t}($.$) is$ a sufficient statistic for the market state.

Fix a rank $x \in[0,1]$. For any such $x$, a rank preserving equilibrium implies $N_{t}(x)$ evolves over time according to the following differential equation:

$$
\begin{aligned}
\dot{N}_{t}(x)= & \lambda\left(s_{t}\right) \widehat{F}\left(x ; s_{t}\right) U_{t}+\gamma\left[1-U_{t}-N_{t}(x)\right] \widehat{\Gamma_{1}}(x) \\
& -\left(\delta+\mu\left[1-\widehat{\Gamma_{0}}(x)\right]+\gamma\left[1-\widehat{\Gamma_{1}}(x)\right]+\lambda\left(s_{t}\right)\left[1-\widehat{F}\left(x ; s_{t}\right)\right]\right) N_{t}(x),
\end{aligned}
$$

where the dot refers to the time derivative $\partial N_{t} / \partial t$ and unemployment $U_{t}=$ $1-N_{t}(1)$. The inflow includes those unemployed who are hired at a firm no greater than $x$ and those employed at a firm with productivity $x^{\prime}>x$ which receives shock $z \leq x$. The outflow contains those whose job is destroyed, who leave to start-up a firm, whose employer enjoys a favorable productivity shock and employees who receive a more favorable outside offer (and so quit). We now formally define equilibrium where $s_{t}=N_{t}($.$) is the aggregate state vari-$ able. We use the following standard notation: that as $N_{t}$ evolves according to the above deterministic process, the wage policy function $w=w(x ; N()$. implies the wage at $\operatorname{rank} x$ evolves according to $w_{t}(x)=w\left(x ; N_{t}\right)$.

Definition Given state $s=N($.$) , a stationary rank preserving equilibrium is$ a wage policy function $w(x ; N()$.$) and hire rate policy h(x ; N()$.$) that$ satisfy

$$
\begin{gathered}
w(x ; N)=b+\lambda(N) \widehat{F}(x ; N) v(x ; N) \\
h(x ; N))=h^{*}(v(x ; N))
\end{gathered}
$$

where

$$
\lambda(N) \widehat{F}(x ; N)=\int_{0}^{x} \frac{h(z ; N) d N(z)}{U+N(z)},
$$

with $U=1-N(1)$. Further along the equilibrium path, $v_{t}(x)=$ $v\left(x, N_{t}().\right)$ and $N_{t}($.$) are solutions to the system of ordinary differential$ 
equations composed of equation (11) together with

$$
\begin{aligned}
& \left(r+\delta+\mu+\gamma+\lambda_{t}\right) v_{t}(x)-\dot{v}_{t}(x) \\
= & p(x)-b+\gamma \int_{0}^{1} v_{t}(z) d \widehat{\Gamma_{1}}(z)+\max _{h \geq 0}\left\langle h v_{t}(x)-c(h)\right\rangle .
\end{aligned}
$$

Finally, an equilibrium is a particular solution to this differential equation system consistent with the initial distribution of employment $N_{0}$ : $[0,1] \rightarrow[0,1]$ and the transversality condition

$$
\lim _{t \rightarrow \infty} v_{t}(x) e^{-r t}=0 \forall x \in[0,1] .
$$

The following mild regularity conditions guarantee that a unique solution exists to the system of ordinary differential equation for every specification of initial conditions:

Assumption 1: $h^{*}(v)$ is Lipschitz continuous. ${ }^{6}$

To identify an upper bound for $v(x ; s)$, suppose in the rip-off strategy that $\lambda(s)=0$ (its employees never quit) and $x=1, \gamma=0$ so that its productivity is always $\bar{p}$. The corresponding maximal payoff $\bar{v}$ is then defined by equation (17) below. To guarantee existence of an equilibrium, we require the following restriction on fundamentals.

Assumption 2: A positive solution for $\bar{v}$ exists to

$$
\bar{v}=\max _{h \geq 0}\left\{\frac{\bar{p}-b+\max _{h \geq 0}\{h \bar{v}-c(h)\}}{r+\delta+\mu}\right\} .
$$

Assumption 2 implies the following upper bound on $h$.

Proposition 3 In any stationary rank-preserving equilibrium, the recruitment rate $h($.$) is bounded above by the effective rate of time discount, i.e.,$ $h(x ; s)<r+\delta+\mu$ for all $(x, s)$.

Proof. By the Envelope Theorem, the RHS of equation (17) is an increasing, convex function of $\bar{v}$ with slope $h^{*}(\bar{v}) /(r+\delta+\mu)$. As the RHS is strictly positive at $\bar{v}=0$ then, given a positive solution exists for $\bar{v}$, it implies $h^{*}(\bar{v})<r+\delta+\mu$. The proposition then follows as $v(x, s) \leq \bar{v}$ for all $x, s$ and $h^{*}($.$) is an increasing function.$

\footnotetext{
${ }^{6}$ Thus, $c(h)$ increasing and strictly convex with the properties $c(0)=c^{\prime}(0)=0$ are sufficient conditions.
} 


\subsection{Homogenous Firms}

Although it is true that the market state $N_{t}($.$) is of infinite dimension in$ the general case, it need not be so in practice. In this section we fully characterize the unique recursive rank-preserving equilibrium in the limiting case of homogenous firms.

In the homogeneous firm case we suppose $p(x)$ is (arbitrarily close to) $\bar{p}$ for all $x$. By construction, each firm $x$ is indifferent to using the rip-off strategy. As the rip-off strategy does not depend on $x$ - the firm posts wage $w=b$ indefinitely - then $v(x ; s)$ cannot depend on $x$ in the limiting homogenous firm case. Let $v_{t}=v\left(x ; s_{t}\right)$ denote the value of an employee in each firm in the limiting case. Optimal recruitment effort $h_{t}=h^{*}\left(v_{t}\right)$ is thus also independent of $x$. The definition of equilibrium then implies the job offer arrival rate reduces to

$$
\lambda_{t}=\lambda\left(s_{t}\right)=h^{*}\left(v_{t}\right) \int_{0}^{1} \frac{d N_{t}(z)}{U_{t}+N_{t}(z)}=-h^{*}\left(v_{t}\right) \ln U_{t} .
$$

Thus conditional on equilibrium hiring intensity $h^{*}\left(v_{t}\right)$, the job offer arrival rate only depends on $N_{t}($.$) through the implied unemployment level U_{t}=$ $1-N_{t}(1)$. Given the limiting case of homogenous firms, we can further restrict the aggregate state vector to $s_{t}=U_{t}$, which is a scalar. Employee value $v_{t}=v\left(U_{t}\right)$ and aggregate unemployment $U_{t}$ evolve according to the pair of autonomous differential equations:

$$
\begin{gathered}
\dot{v}_{t}=\left(r+\delta+\mu-h^{*}\left(v_{t}\right) \ln U_{t}\right) v_{t}-\left(\bar{p}-b+\max _{h \geq 0}\left[h v_{t}-c(h)\right]\right) \\
\dot{U}_{t}=\delta(1-U)-\left[\mu-h^{*}\left(v_{t}\right) \ln U_{t}\right] U_{t} .
\end{gathered}
$$

The solution of interest, $v=v(U)$, thus solves the differential equation

$$
\frac{d v}{d U}=\frac{\dot{v}}{\dot{U}}=\frac{\left(r+\delta+\mu-h^{*}(v) \ln U\right) v-\left(\bar{p}-b+\max _{h \geq 0}\{h v-c(h)\}\right)}{\delta-[\delta+\mu-h(v) \ln U] U} .
$$

It is well known that a unique continuous solution exists to this equation for all $U \in[0,1]$ if and only if the ODE system composed of (19) and (20) has a unique steady state solution and the steady state is a saddle point. Indeed, the branch of the saddle path that converges to the steady state for every initial value of aggregate unemployment is the equilibrium value of a match function. Below we prove that these necessary and sufficient conditions hold. 
Any steady state solution is the $(U, v)$ pair defined by the pair of equations

$$
\begin{aligned}
\delta-(\mu+\delta) U & =-h^{*}(v) U \ln U \\
\left(r+\delta+\mu-h^{*}(v) \ln U\right) v & =\bar{p}-b+\max _{h \geq 0}\{h v-c(h)\}
\end{aligned}
$$

We first show there exists a single solution pair $(v, U)$ to these equations.

Equation (21) describes the $\dot{U}=0$ locus drawn in Figure 1 below. The LHS of (21) is zero at $U=\frac{\delta}{\delta+\mu}<1$ and decreases at the constant rate $\delta+\mu$. For any $v>0$, the RHS is zero at $U=0,1$, is positive and strictly concave in $U$ for $U \in(0,1)$. Hence a unique, positive value of $U$ strictly less than $\delta /(\mu+\delta)$ exists for every positive value of $v$. As $h^{*}($.$) is an increasing$ function, it follows that $U$ decreases as $v$ increases with limiting properties $U \rightarrow \delta /(\mu+\delta)$ as $v \rightarrow 0$ and $U \rightarrow 0$ as $v \rightarrow \infty$.

Equation (22) describes the $\dot{v}=0$ locus in Figure 1. The RHS does not depend on $U$, is strictly positive at $v=0$ and, for $v \in[0, \bar{v}]$, the Envelope Theorem implies it is a strictly increasing function of $v$ with slope $h^{*}(v)<$ $r+\mu+\delta$ [Proposition 3]. The LHS is instead zero at $v=0$ and is a strictly increasing function of $v$ with slope strictly greater than $r+\mu+\delta$. Thus if a solution exists to equation (22) it must be unique. Note further that at $U=1$, the unique solution is $v=\bar{v}$. As the LHS is decreasing in $U$, it follows that a solution for $v \in[0, \bar{v}]$ exists for all $U \in[0,1]$ where $v$ increases as $U$ increases with limiting properties $v \rightarrow 0$ as $U \rightarrow 0$ and $v=\bar{v}$ at $U=1$. Continuity now implies a unique steady state solution for the pair $(v, U)$ exists and steady state $U \in[0, \delta /(\mu+\delta)]$.

The dynamics implied by the ODE system composed of (20) and (19) are illustrated by its phase diagram portrayed in Figure 1. The intersection of the two singular curves is a saddle point that attracts a unique converging saddle path from any initial value of $U$. Finally, because the growth rate in $v$ on the unstable path above the saddle path must eventually exceed the rate of interest, while the unstable path below the steady state ultimately yields zero $v$ (which contradicts optimal firm behavior), the stable path represents the only Markov equilibrium.

Proposition 4 A unique stationary rank-preserving equilibrium exists in the limiting case of equally productive firms. Further equilibrium $v(U)$ increases with unemployment. 
Figure 1: Phase Diagram 
The phase diagram and the equilibrium adjustment path have a natural interpretation as a dynamic supply and demand model for match formation. Of course, the value of an employee $v$ is the relevant price. A higher employee value induces greater recruiting effort which yields greater utilization of the available labor force by reducing unemployment duration, a relationship represented by the downward sloping $\dot{U}=0$ singular (supply) curve. However, the (demand) price $v$ increases at the margin with $U$ along the $\dot{v}=0$ curve because lifetime wages decrease with aggregate unemployment. Along the optimal adjustment path, the price adjusts in response to "excess demand" to bring the supply and demand prices into balance.

Note that any common and unanticipated positive shock to the productivity of a match $p$ shifts up the dynamic "demand" curve [the $\dot{v}=0$ curve] in Figure 1 but has no direct effect on "supply." The result is an increase in the steady state value of employment $(v)$ and a decrease in unemployment $(U)$ as in the canonical search and matching model. Hence, the equilibrium value of $v$ jumps up initially and then adjusts slowly downward along the path converging to the new steady state value.

It is straightforward to back out equilibrium micro-behavior: the equilibrium wage paid by a rank $x$ firm is

$$
w(x ; U)=b+h^{*}(v) v \ln \left(\frac{U+N(x)}{U}\right)
$$

with $v=v(U)$. This expression confirms that $w($.$) is indeed rank preserving,$ where $w(0 ; U)=b$ is the lowest wage paid. Although equilibrium $v(U)$ is independent of $N($.$) , this is not the case for the induced wage dispersion w($.$) .$ Thus even at steady state $U$, wage dispersion (and quit turnover) dynamics are non-trivial. Furthermore there are additional composition effects if $N_{t}($. lies away from its steady state.

The equilibrium quit rate is

$$
q(W(x ; U) ; U)=-h^{*}(v) \ln [U+N(x)]
$$

which is decreasing in $x$, being $-h^{*}(v) \ln U$ at $x=0$ (the bottom rank firm) and zero at $x=1$. Note a firm's equilibrium quit rate depends directly on the level of unemployment. This occurs as firms are more likely to recruit from the pool of unemployed workers the larger is that pool. As each quit costs the firm $v$ in continuation payoff, each firm is thus better off with higher unemployment as competing firms are then less likely to poach one of its 
employees. Indeed it is this poaching externality which explains why, outside of steady state, equilibrium $v($.$) is increasing in U$.

Wage dispersion arises as hiring is costly and firms offer different wages to reduce their employee quit rates. As in BM, the wages offered are ranked by productivity $x$ where higher ranked firms pay higher wages and enjoy lower quit rates. Unlike BM, however, there is no simple correlation between wages and firm size. Here instead high rank $x$ firms enjoy high expected growth rates equal to $h^{*}(v)[1+\ln [U+N(x)]]$. Thus a firm's growth rate is independent of its size $n$, depending only on its productivity rank $x$ within the set of all competing firms, the level of unemployment, where $v=v(U)$, and on the distribution of firm sizes $N($.$) . The expected growth rate of employment$ depends only on whether or not $U$ exceeds its steady state value. Across surviving firms, firms with $x$ satisfying $U+N(x)>1 / e \simeq 0.37$ have positive expected growth rates, while lower rank firms have negative growth rates. Current firm size $n(x, t)$ thus evolves according to a geometric Markov process where currently large firms will typically have existed for a longer time and enjoyed higher than average growth rates.

\subsection{Heterogeneous Firms}

This section now generalizes the analysis to a finite number of firm types. Let $p_{i}$ represent the productivity of firms of type $i=1, . ., I$; i.e. $p(x)=p_{i}$ for all $x \in\left(x_{i-1}, x_{i}\right] \subseteq[0,1]$ where the set $\left(x_{i-1}, x_{i}\right]$ represents the set of firms of type $i$ and $x_{0}=0, x_{I}=1$. As the value of an employee is the same for all firms of the same type, let $v_{i}(s)=v(x ; s)$ for $x \in\left(x_{i-1}, x_{i}\right]$, $i=1,2, \ldots I$, denote the value of an employee in type $i$ firms in aggregate state $s . \mathbf{v}=\left(v_{1}, v_{2}, \ldots, v_{I}\right)$ denotes the corresponding vector of employee values. Let $N_{i}=N\left(x_{i}\right)$ denote the number of workers employed in firms of type $i$ or less and $\mathbf{N}=\left(N_{1}, N_{2}, \ldots, N_{I}\right)$ denotes the corresponding vector. Note unemployment $U=1-N_{I}$. Recall that $\widehat{\Gamma_{1}}\left(x_{i}\right)-\widehat{\Gamma_{1}}\left(x_{i-1}\right)$ describes the probability the of productivity shock $z \in\left[x_{i-1}, x_{i}\right]$. With no loss of generality we set $\widehat{\Gamma_{1}}\left(x_{i}\right)=x_{i}$ so that the set $\left\{x_{i}\right\}$ describe the probability distribution of type $i$ shocks. For simplicity, suppose all new start-ups have initial productivity $p=p_{I}$.

With finite types, we describe equilibrium where $s=\mathbf{N}$ describes the 
relevant aggregate state vector. In this case, equation (14) implies

$$
\begin{aligned}
\lambda_{i}(\mathbf{v} ; \mathbf{N}) & \equiv \lambda \widehat{F}\left(x_{i} ; \mathbf{N}\right)=\sum_{j=1}^{i}\left[\int_{x_{j-1}}^{x_{j}} h^{*}\left(v_{j}\right) \frac{d N(z)}{1-N_{I}+N(z)}\right] \\
& =\sum_{j=1}^{i} h^{*}\left(v_{j}\right) \ln \left(\frac{1-N_{I}+N_{j}}{1-N_{I}+N_{j-1}}\right), i=1,2, \ldots I .
\end{aligned}
$$

Letting $\lambda(\mathbf{v} ; \mathbf{N})=\lambda_{I}(\mathbf{v} ; \mathbf{N})$ denote the equilibrium job offer arrival rate, equation (15) can then be written as

$$
\begin{aligned}
\dot{v}_{i}= & (r+\delta+\mu+\gamma+\lambda(\mathbf{v} ; \mathbf{N})) v_{i} \\
& -\left(p_{i}-b+\gamma \sum_{j} v_{j}\left[x_{j}-x_{j-1}\right]+\max _{h \geq 0}\left\langle h v_{i}-c(h)\right\rangle\right) .
\end{aligned}
$$

The laws of motion for the distribution of employment over types $i$ are described by:

$$
\begin{aligned}
\dot{N}_{i}= & \lambda_{i}(\mathbf{v} ; \mathbf{N})\left[1-N_{I}\right]+\gamma\left[N_{I}-N_{i}\right] x_{i}+\mu \Gamma_{0 i} \\
& -\left(\delta+\mu+\gamma\left[1-x_{i}\right]+\sum_{j>i} h^{*}\left(v_{j}\right) \ln \left(\frac{1-N_{I}+N_{j}}{1-N_{I}+N_{j-1}}\right)\right) N_{i},
\end{aligned}
$$

where $\Gamma_{0 i}=0$ for $i<I$ and $\Gamma_{0 I}=1$ (i.e. new firms have initial type $i=I$ ).

Given Assumption 1, the right hand sides of the above differential equations (24),(25) are Lipschitz continuous provided that $N_{I}<1$. Thus for all $\mathrm{N}$ satisfying

$$
\frac{1-N_{I}+N_{j}}{1-N_{I}+N_{j-1}}>0
$$

the system of ODE has a unique solution for every set of initial conditions satisfying the inequality.

For a stationary (Markov) equilibrium, we seek a particular solution to the system that can be represented as a function $\mathbf{v}(\mathbf{N})$ that map the set of employment distributions into the feasible set of vector values given that map is used to solve for the evolution of the distribution of employment. An equilibrium, then, is a fixed point of the transformation $M$ defined by the forward solutions to (24), expressed here as

$$
\begin{aligned}
(M v)_{i}\left(\mathbf{N}_{0}\right)= & \int_{0}^{\infty}\left(\begin{array}{c}
p_{i}-b+\gamma \sum_{j} v_{j}\left(\mathbf{N}_{t}\right)\left[x_{j}-x_{j-1}\right] \\
+\max _{h \geq 0}\left\langle h v_{i}\left(\mathbf{N}_{t}\right)-c(h)\right\rangle
\end{array}\right) \\
& \times e^{-\int_{0}^{t}\left(r+\delta+\mu+\gamma+\lambda\left(\mathbf{v}\left(\mathbf{N}_{\tau}\right), \mathbf{N}_{\tau}\right)\right) d \tau} d t
\end{aligned}
$$


where $\mathbf{N}_{t}$ is the backward solution to the laws of motion for the employment distribution, equation (25), conditional on the given initial distribution of employment over firm types. That is

$$
\begin{aligned}
(M N)_{i t}\left(\mathbf{N}_{0}\right)= & N_{i 0} e^{-\int_{0}^{t} \alpha_{i}\left(\mathbf{v}_{\tau}, \mathbf{N}_{\tau}\right) d \tau} \\
& +\int_{0}^{t}\left[\begin{array}{c}
\lambda_{i}\left(\mathbf{v}_{t} ; \mathbf{N}_{t}\right)\left[1-N_{I \tau}\right] \\
+\gamma x_{i} N_{I \tau}+\mu \Gamma_{0 i}
\end{array}\right] e^{-\int_{\tau}^{t} \alpha_{i}\left(\mathbf{v}_{z}, \mathbf{N}_{z}\right) d z} d \tau, \\
\text { where } \alpha_{i}(\mathbf{v}, \mathbf{N})= & \delta+\mu+\gamma+\sum_{j>i} h^{*}\left(v_{j}\right) \ln \left(\frac{1-N_{I}+N_{j}}{1-N_{I}+N_{j-1}}\right)
\end{aligned}
$$

Any function $\mathbf{v}(\mathbf{N})$ that is a fixed point of the map $(M v)$ is a recursive stationary equilibrium. As it maps the set of Lipschitz continuous functions into itself and this set is equi-continuous, closed and convex, existence of a fixed point follows by Schauder's theorem if the set is also bounded in the supnorm.

Proposition 5 With a finite number of firm types, a stationary rank-preserving equilibrium exists if there is positive initial unemployment; i.e. $U=1-N_{I 0}>$ 1.

Proof. To complete the proof, we need only show that $M$ maps the set of Lipschitz continuous functions that are bounded in supnorm into itself. We consider the bound $\bar{v}$ defined by Assumption 2 and $\bar{N}$ defined by

$$
\bar{N}=\max \left\langle N_{I 0}, \frac{\mu+h^{*}(\bar{v}) \ln \left(\frac{1}{1-\bar{N}}\right)}{\delta+\mu+h^{*}(\bar{v}) \ln \left(\frac{1}{1-\bar{N}}\right)}\right\rangle .
$$

Note that $\bar{N}<1$ if $N_{I 0}<1$.

Let $\|f\|=\sup _{f \in \mathcal{F}}|f|$ represent the supnorm. As $\lambda_{i}\left(\mathbf{v}_{t}, \mathbf{N}_{t}\right) \leq \lambda\left(\mathbf{v}_{t}, \mathbf{N}_{t}\right)$ for all $i$, and $0 \leq M N_{i t} \leq M N_{I t}$ if $0 \leq N_{i t} \leq N_{I t}$, it is sufficient to show that $(M v)_{i}\left(\mathbf{N}_{0}\right) \leq \bar{v}$ and $(M N)_{I t}\left(\mathbf{N}_{0}\right) \leq \bar{N}<1$.

Now for $i=I$ the law of motion for $N_{I}$ reduces to

$$
\dot{N}_{I}=[\mu+\lambda(\mathbf{v} ; \mathbf{N})]\left[1-N_{I}\right]-\delta N_{I}
$$

which yields the more convenient mapping:

$$
(M N)_{I t}\left(\mathbf{N}_{0}\right)=N_{I 0} e^{-\int_{0}^{t} \alpha\left(\mathbf{v}_{\tau}, \mathbf{N}_{\tau}\right) d \tau}+\int_{0}^{t}[\mu+\lambda(\mathbf{v} ; \mathbf{N})] e^{-\int_{\tau}^{t} \alpha\left(\mathbf{v}_{z}, \mathbf{N}_{z}\right) d z} d \tau,
$$

where $\alpha(\mathbf{v}, \mathbf{N})=\delta+\mu+\lambda(\mathbf{v} ; \mathbf{N})$. 
Further if $v_{i}\left(\mathbf{N}_{t}\right) \leq \bar{v}$ and $N_{i} \leq \bar{N}$ for all $i$, then

$$
\begin{aligned}
\lambda(\mathbf{v} ; \mathbf{N}) & \equiv \sum_{j} h^{*}\left(v_{j}\right) \ln \left(\frac{1-N_{I}+N_{j}}{1-N_{I}+N_{j-1}}\right) \\
& \leq h^{*}(\bar{v}) \sum_{j=1}^{I} \ln \left(\frac{1-N_{I}+N_{j}}{1-N_{I}+N_{j-1}}\right) \\
& =h^{*}(\bar{v}) \ln \left(\frac{1}{1-N_{I}}\right) \\
& \leq h^{*}(\bar{v}) \ln \left(\frac{1}{1-\bar{N}}\right) \equiv \bar{\lambda}
\end{aligned}
$$

Hence with $\lambda_{t}=\lambda\left(\mathbf{v}_{t} ; \mathbf{N}_{t}\right)$ :

$$
\begin{aligned}
\left\|(M N)_{i t}\left(\mathbf{N}_{0}\right)\right\| & \leq\left\|(M N)_{I t}\left(\mathbf{N}_{0}\right)\right\| \\
& =\sup _{t \geq 0}\left\{N_{I o} e^{-\int_{0}^{t}\left(\delta+\mu+\lambda_{\tau}\right) d \tau}+\int_{0}^{t}\left[\lambda_{\tau}+\mu\right] e^{-\int_{\tau}^{t}\left(\delta+\mu+\lambda_{z}\right) d z} d \tau\right\} \\
& =\sup _{t \geq 0}\left\{\begin{array}{c}
N_{I o} e^{-\int_{0}^{t}\left(\delta+\mu+\lambda_{\tau}\right) d \tau} \\
+\int_{0}^{t} \frac{\lambda_{\tau}+\mu}{\delta+\mu+\lambda_{\tau}}\left(\delta+\mu+\lambda_{\tau}\right) e^{-\int_{\tau}^{t}\left(\delta+\mu+\lambda_{z}\right) d z} d \tau
\end{array}\right\} \\
& \leq \sup _{t \geq 0}\left\{\begin{array}{c}
N_{I o} e^{-\int_{0}^{t}\left(\delta+\mu+\lambda_{\tau}\right) d \tau} \\
+\frac{[\bar{\lambda}+\mu]}{(\delta+\mu+\bar{\lambda})} \int_{0}^{t}\left(\delta+\mu+\lambda_{\tau}\right) e^{-\int_{\tau}^{t}\left(\delta+\mu+\lambda_{z}\right) d z} d \tau
\end{array}\right\}
\end{aligned}
$$

where $\bar{\lambda}$ is an upper bound on $\lambda_{t}$. Integration now yields:

$$
\begin{aligned}
\left\|(M N)_{I t}\left(\mathbf{N}_{0}\right)\right\| & \leq \sup _{t \geq 0}\left\{\begin{array}{c}
N_{I o} e^{-\int_{0}^{t}\left(\delta+\mu+\lambda_{\tau}\right) d \tau} \\
+\frac{[\bar{\lambda}+\mu]}{(\delta+\mu+\bar{\lambda})}\left[1-e^{-\int_{0}^{t}\left(\delta+\mu+\lambda_{\tau}\right) d \tau}\right]
\end{array}\right\} \\
& =\max \left\langle N_{I 0}, \frac{\bar{\lambda}+\mu}{\delta+\mu+\bar{\lambda}}\right\rangle=\bar{N}<1 .
\end{aligned}
$$

Furthermore:

$$
\begin{aligned}
\left\|(M v)_{i}\left(N_{0}\right)\right\| & \leq \int_{0}^{\infty}\left(\begin{array}{c}
\bar{p}-b+\gamma \bar{v} \\
+\max _{h \geq 0}\{h \bar{v}-c(h)\}
\end{array}\right) e^{-\int_{0}^{t}\left(r+\delta+\mu+\gamma+\lambda_{\tau}\right) d \tau} d t \\
& \leq \frac{\bar{p}-b+\max _{h \geq 0}\{h \bar{v}-c(h)\}+\gamma \bar{v}}{r+\delta+\mu+\gamma} \\
& =\bar{v}<\infty
\end{aligned}
$$


by definition of $\bar{v}$ and Assumption 2 .

\section{Conclusion.}

We have shown the introduction of a hiring margin into the matching framework with on-the-job search yields a surprisingly rich and tractable equilibrium framework. We have fully characterized and established the existence of rank-preserving equilibria in dynamic, non-steady state economies. The environment considered is particularly rich. There is turnover of firms with innovative start-up companies replacing existing firms which suffer firm destruction shocks. There is quit turnover where, in equilibrium, workers quit low productivity firms to take employment in high productivity firms. Even with no precommitment by firms on future wages, equilibrium wage dispersion arises as higher productivity firms are willing to pay a higher wage to reduce employee quit rates. Furthermore firm growth rates are size independent: high productivity firms pay higher wages, enjoy low quit rates, recruit new employees with greater effort and so enjoy a positive expected growth rate. The converse is the case for low productivity firms. The structure also allows for firm specific productivity shocks, so that previously successful firms may ultimately decline should they receive an unfavorable productivity draw.

The characterization of equilibrium is particularly simple in the limiting case of equally productive firms. Even though the distribution of firm sizes is infinitely dimensional, equilibrium aggregate behavior depends only on the level of unemployment. A particularly useful insight is that the value of a firm is increasing in the level of unemployment. This occurs as, with higher unemployment, firms are less likely to poach each others' employees. As a quit is costly to the firm (it is costly to hire a replacement), this poaching externality implies an employee is more valuable (more likely to stay) as unemployment increases. As greater employee value implies firms respond by increasing recruitment effort, the equilibrium dynamics of the economy are intrinsically stable.

This new rich and tractable framework opens up several important directions for future research. The equally productive firms case is important as equilibrium dichotomizes into (i) macroeconomic behavior where, depending only on the level of unemployment $U$, equilibrium determines gross job creation rates and (ii) microeconomic behavior where wages and quit turnover 
at the firm level depends on a firm fixed effect $x$, the collective recruitment effort of firms (determined in the macroequilibrium) and the distribution of firm sizes which itself evolves endogenously over time. Given the Markov structure of the model, it is clear the model will generalize to a framework where aggregate productivity $p$ and job destruction parameter $\delta$ evolve according to a stochastic Markov process. The extension is interesting not only because firms use optimal wage setting strategies, rather than Nash bargaining, but also because the insights of Coles and Moghaddasi (2011) suggest this framework will fit the business cycle volatility and persistence data as described in Shimer (2005). Indeed the model will automatically generate procyclical quit turnover: high aggregate productivity $p$ will increase firm hiring rates, thus increasing worker quits from the lower end of the type xdistribution. Furthermore periods of high unemployment will have lower quit rates as newly available jobs are more likely to be filled by the unemployed.

An important distinction between this paper and the BM approach is that in the latter framework the wage had to do two jobs: wages were used both to attract new employees and to retain existing employees. Here instead the hiring margin is fully targeted by recruitment strategy, leaving wages to target only the quit margin. The resulting equilibrium wage structure is different. Preliminary work suggests the properties of the wage offer density are more akin to the data. Furthermore the employment weighted distribution of wages, a distribution which is only stationary in the steady state, can be quite complex.

The equilibrium wage structure considered here relies on a reputation effect: that if the firm ever announces a low wage, its employees forever anticipate the rip-off wage $w=b$ in the future. It is well known in infinitely repeated games that allowing reputation effects can support a multiplicity of equilibria. The same is true here. The key is that if employees forever expect wage $w=b$ in the future, then paying a higher wage for an instant $d t>0$ has (almost) no effect on turnover. Thus given those beliefs it is optimal simply to pay $w=b$. An alternative construction, then, is that the high wage strategy $w(x, n ; s)$ may instead yield strictly greater profit than the rip-off strategy. The wage path $w($.$) remains credible as any deviation is$ punished by employee expectations $w=b$ in the entire future (and is thus less profitable). ${ }^{7}$ The approach taken here, however, is not only more tractable (it describes a Markovian wage process), we believe it is the more persuasive

\footnotetext{
${ }^{7}$ we are grateful to Ludo Vischers for pointing out this possibility
} 
case for two reasons.

First for the equal productivity case, the alternative approach implies a firm exists with $x^{*}>0$ which makes strictly greater profit than does firm $x=0$ (which pays $w=b$ ). Firm $x=0$ would clearly like to adopt the wage policy $w\left(x^{*} ; s\right)$ as this yields greater profit. Further its employees would also be better off if its firm did adopt this wage policy (which is different to, and thus more generous than the rip-off strategy). The only reason the firm does not do this, however, is because its employees insist on expecting wage $w=b$ in the entire future. Although consistent with subgame perfect behavior, this outcome does not seem compelling: it seems more reasonable that the firm and its employees should coordinate (renegotiate) to the jointly preferred $x^{*}$ wage policy. Note that the equilibrium structure above rules out jointly preferred wage policies - workers strictly prefer a higher $x$ wage policy $w(x ; s)$, while firms do not. All firms, being indifferent to the same rip-off strategy, enjoy the same equilibrium payoff.

Second, a different approach is to introduce asymmetric information: that firms know their productivity $p(x)$ but workers only observe a history of wages paid. Indeed there is a large wage bargaining literature based on this assumption. Preliminary work (with wage setting by firms) identifies a fully revealing equilibrium where higher productivity firms announce strictly higher wages and so enjoy strictly lower employee quit rates. Further in the limit as productivity dispersion disappears, each firm $x$ makes the same expected profit. This limiting equilibrium (with asymmetric information) has properties very similar to the one identified above.

\section{References}

[1] Burdett, K, and K Judd (1983) "Equilibrium Price Dispersion," Econometrica, 51: 955-969.

[2] Burdett, K and D T Mortensen (1998). "Wage Differentials, Employer Size and Unemployment," International Economic Review 39: 257-273.

[3] Coles, M G (2001) "Equilibrium Wage Dispersion, Firm Size and Growth," Review of Economic Dynamics, vol. 4(1), pages 159-187.

[4] Lucas, R (1967). "Adjustment Costs and the Theory of Supply," Journal of Political Economy (74): 321-334. 
[5] Merz, M, and E Yashiv (2007). "Labor and the Market Value of the Firm," American Economic Review 90: 1297-1322.

[6] Menzio, G, and S Shi (2010). "Directed Search on the Job, Heterogeneity, and Aggregate Fluctuations," American Economics Review 100: 327-332.

[7] Moscarini, G and F Postel-Vinay (2010). "Stochastic Search Equilibrium," Yale working paper. 